\title{
Etymologische Bemerkungen
}

Zur Etymologie des ungarischen Ableitungssuffixes -ság, -ség.

Mit dem ungarischen denominalen Ableitungssuffix -ság, -ség werden Eigenschaftsnamen und Kollektivwörter gebildet, z. B. jóság 'Güte' ( jó 'gut'), szépség 'Schönheit' ( szép 'schön'), katonaság 'Militär, Soldaten, Soldatenvolk' ( katona 'Soldat'), népség 'Völkerschaft, Volk' ( nép 'Volk'). Von den zwei Formen ist die vordervokalische ursprünglich, und das Suffix ist auf dem Wege der Agglutination aus einem selbständigen Worte entstanden. ZoLTÁx GoмBocz führt in seiner Untersuchung über den Ursprung dieses Suffixes, "A ság-ség képző eredete» (s. Gombocz Zoltán összegyüjtött müvei. I, S. 153-156), reichliche Beispiele für es aus der alten Literatur an. Nach Gombocz kommt es zum erstenmal in der Leichenrede (Halotti Beszéd) vor; es ist schon damals mit dem Hauptwort zusammengeschrieben und weist in Verbindung mit hintervokalischen Wörtern den Vokal a auf: uimadsag-, birsag-, uruzag-. Aber die alten Sprachdenkmäler enthalten auch Beispiele, bei denen das Suffix getrennt geschrieben ist und bei denen es auch in Verbindung mit hintervokalischen Wörtern noch den Vokal $e$ hat; die letzten Fälle dieser|Art stammen aus der Zeit gegen 1470: alkolmasseg (vgl. das heutige alkalmasság 'Tauglichkeit'), alazatus segheyerth (lies: alázatus ségéjért) $=$ 'humilitatis affectu'. Anfänglich bildete -ság, -ség Eigenschaftsnamen, von den Kollektivwörtern liegen aus dem XVI. - XVII. Jahrhundert noch wenige Beispiele vor (s. des Näheren Gombocz a. a. O.). Gombocz nimmt denn auch an, dass es im Urungarischen ein Substantiv *ségi 〜 *ségü (?*sëgi $\sim$ *sëgü) gab, das eine abstrakte Bedeutung hatte und das 
schon vor der Zeit der Leichenrede zu dem in Rede stehenden Suffix wurde; dieses Wort kann nach ihm keine türkische oder ossetische Entlehnung sein, sondern gehört zu dem alten finnisch-ugrischen Erbgut der ungarischen Sprache.

Für -ság, -ség hat man zwei Etymologien aufgestellt. Die eine stammt von J. Budenz. Da in einigen finnisch-ugrischen Sprachen Wörter, die 'Zeit' bedeuten, auch zur Bildung von Eigenschaftsnamen dienen [z. B. syrj. (Wichm. -Uot.) lun 'Tag': ozirlun 'Reichtum' ozir 'reich'; mord. (Paas.) tši, š 'Sonne; Tag': šumbra-tši usw. 'Gesundheit '〜 šmbra usw. 'ganz, unversehrt, gesund'], vermutete Budenz in Nyr. I $345-349$, dass auch ung. *ség ursprünglich 'eine Art Zeit' bedeutet hätte und dass seine Entsprechungen das soeben erwähnte mord. tšs, ši 'Sonne; Tag' und fi. (Renv.) sää 'tempestas, variatio tempestatis', 'Witterung' wären. BERnHARD MUNKácsi hat zu diesen noch aus dem Wogulischen das Substantiv T čou, šou, K śou, śö u, L śi, ši 'Zeit' gestellt (s. NyK XXII 16, KSz. II 41). Die spätere Forschung hat diese Zusammenstellungen jedoch verworfen: für das fi. Wort hat man andere Entsprechungen aufgestellt (s. z. B. E. N. SEtäLÄ Festskrift til Vilhelm Thomsen 230, 236, FUF II 255, H. PAAsoner s-Laute 130-132, YRJö WichmanN FUF XVI 204, J. Szinnyei NyH7 140, Y. H. Tolvonen Suomi V: 16, S. $305-$ $306)$; wog. čou, śi usw. hält SzInNyei $\mathrm{NyH}^{3} 105$ für eine Entlehnung aus dem tatarischen Wort, das nach Bálint folgendermassen lautet: čak 'Zeit, Mass', bu čakta, bu čarinda 'gegenwärtig, heut zu Tage', čakl 'mässig; soviel wie; (c. dat.) bis' usw. (s. auch Paasonen FUF VI 238), und das mordwinische Wort wiederum hat ursprünglich mit $k$ angelautet und gehört u. a. mit fi. kehä 'errichtetes Hausgezimmer; Umkreis; Hof um Sonne od. Mond', wog. kuš, kis 'Reif' usw. zusammen (s. Paasonen FUF VI 238-239, Toivonen FUF XIX 64-65).

Die andere Etymologie stammt von Gombocz. Da er unter den 'Zeit' bedeutenden Wörtern der verwandten Sprachen kein einziges fand, das sich mit ung. -ság, -ség zusammenstellen liesse, nahm er in der obenerwähnten Untersuchung an, dass dieses ursprünglich 'Schattenseele' bedeutet hätte und dass es mit folgenden von Paasonen in JSFOu. XXVI $\mathrm{X}_{4}$ S. 10-17 
verbundenen Wörtern verwandt sei: ostj. (Karj.-Toiv.) Koš.,

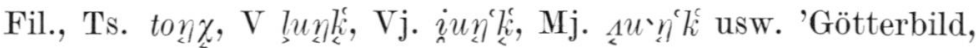
Götzenbild; Schutzgeist usw.', wog. (Kann. Vir. 1933 S. $419-$ 420, Wog. Volksd. I 409) KM $t \curvearrowleft \beta, \operatorname{tn} \tilde{\beta} \beta i$ 'Schemen- oder Schattenseele des Menschen', KO teß, P teßs's, syrj. šön 'Schatten

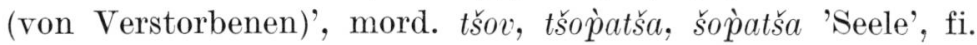
hии 'gast, spöke, vålnad'; in diesen war der anlautende Konsonant $* s$, der inlautende $* \eta$. Diese Zusammenstellung kann nicht richtig sein, denn sie enthält einige schwere Fehler. Erstens entspricht, wie schon Gombocz selbst hervorhebt, der ungarische vordere Vokal nicht der Erwartung, und zweitens passt ung. $s$ - nicht als Vertreter des fiugr. * $\xi^{-}$, denn $* s$ - ist im Ungarischen bekanntlich geschwunden; eine Ausnahme hat man allerdings in dem Worte ung. savanyú 'sauer' $\sim$ ostj.

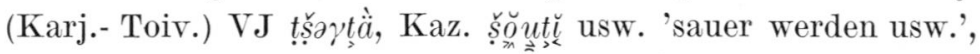
wog. (Kann. Vok. 201) VN, VS, LU šể -, LO sāß- 'säuern' usw., tscher. šà pầ 'sauer', mord. E tša p̀amo, M šà̀amă 'sauer', fi. hapan id. (s. z. B. Szinnyei $\mathrm{NyH}^{7} 27$ ) sehen wollen, aber wie Paasonen FUF XII 300- und Toivonen FUF XX 136138 gezeigt haben, muss man auch in ihm von der Affrikata *tš ausgehen. ${ }^{1}$ Ausserdem ist die Zusammenstellung von Gombocz semasiologisch nicht gelungen.

Wie oben bemerkt wurde, hat Munkácsi wog. čou, śi usw. 'Zeit' u. a. mit ung. -ság, -ség verglichen. Diese Zusammenstellung verdient, trotz der entgegengesetzten Ansicht Szinynyeis, ernste Beachtung, und im Folgenden wird denn auch die Frage einer eingehenden Betrachtung unterworfen.

Die Veröffentlichungen Munkácsis bieten über das wogulische Wort folgende Angaben: (Munk.- Szil., Munkácsi VNGy. II 512, NyK XXII 16, 61, XXIII 379, XXIV 19, 317) T $\check{c} \theta$, šou, K śou, śöu, P, LM śi, LU ši 1) 'Zeit': 'T ti-šsut 'ekkor', T tu-šout, K ton-śöut, P ton-śit, LM tä-šit 'damals' (ti 'dieser', ton, tä 'jener'); LM äkw'-mät-śit 'einmal', K äkwé-mäiśt id. (äkw'-mät 'egy valamelyik, egyik'); K khotèl=jot-śit 'délkor,

1 Ung. sasanyú ist ausserdem als eine Ableitung des Wortes só 'Salz' erklärt worden, s. J. Tequert MNy. XXVII $111-118$ und Géza BÁrczi, Magyar Szófejtö Szótár S. 267. 
dél idején', LM khwal-śit 'reggeli időben' (khotèl=jot 'Mittag', khwal 'der Morgen'); P soat-śi 'eine Woche', LM saat-śi id. (soat 'Woche'), K jōñkhèp-śöu 'egy hónapnyi ideig' (jōñlhèp 'Monat'); P lū jel-ōrtènät-śit 'wenn das Pferd müde wird', LM nal-pätèm śiänt 'als sie herunterkamen'; 2) 'ein gewisses Mass, eine gewisse Menge': K nè-śou, P män-śi, LU män-ši

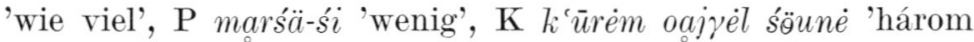
versztnyire', T kitä $\chi$ täl šsu 'zwei Klafter weit'; 3) 'Art': LM äm śi khum 'ein Mann meines Schlages', äm śiim poj, täu śiät poj 'so reich wie ich, wie er', $\mathrm{P}$ okw' śi khumei 'egyforma emberek', LM äkw' śikwèl joami 'gleicherweise fortschreiten', K tète śöul 'auf diese Art'. Nach den handschriftlichen Sammlungen ARTturi Kannistos lautet das Wort so: TJ, TČ

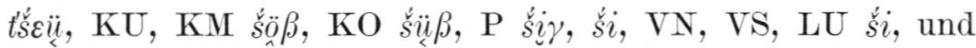
die Bedeutung ist dieselbe wie bei Munkácsi.

Wie oben erwähnt wurde, wäre wog. č ou, sii nach Szinnyei dem tatarischen Worte čak 'Zeit, Mass' entlehnt. Das Lautgewand macht jedoch die Annahme einer solchen Entlehnung schwierig. Erstens muss man sich nämlich erinnern, dass dem tatarischen inlautenden $k$ (und in den meisten Fällen auch dem $\gamma_{k}$ ) im Wogulischen im Silbenanlaut $k, k$, im Silbenauslaut $\chi, \chi$ (also nicht $\gamma, \beta$ ) entspricht (s. des Näheren Kannisto Tat. Lehnw. 12-13, 15-16). Zweitens ist von dem Vokal des wogulischen Wortes zu beachten, dass KU, KM $\ddot{o}$ und $\mathrm{KO} \ddot{\ddot{~}}$ offenbar unter der Einwirkung des nachfolgenden $\beta$ aus einem früheren * entstanden sind; ein interdialektischer Wechsel $\gamma$ $\sim \beta$ ist nämlich neben illabialen Vokalen auch sonst nicht

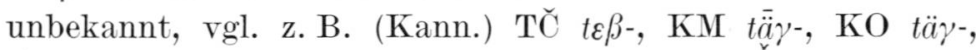

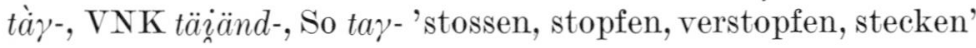

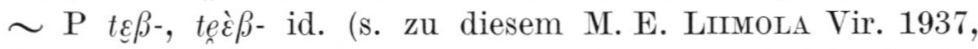
S. 167 , T. E. Uotila FUF XXVI, S. 178). Die Vokale der verschiedenen Dialekte haben also früher untereinander eine solche Reihe gebildet, die in manchen bodenständigen Wörtern begegnet (Beispiele s. bei Kannisto Vok. 45, 53-). Diese Vokalserie passt aber nicht zur Entsprechung des tat. $a$, denn in den tatarischen Lehnwörtern ist das a des Originals durch die Vokale der *a bzw. *a-Serien (also durch $a$, å resp. $\bar{a}, \bar{a}$, $\bar{o}, \overline{\dot{o}}, \bar{n})$ vertreten, dialektweise auch durch die Vokale der $*_{0-}$ 
Serie und bisweilen durch diejenigen der $* \bar{a}$, *a-Serie (s. Kannisto Tat. Lehnw. 21-24). Ausnahmsweise begegnet in KU $\grave{a}_{o}$, $\ddot{a} \grave{a}_{o}$, das in den einheimischen Wörtern auf urwog. * $* \bar{a}$ zurückgeht, gegenüber dem tatarischen $a$ in folgenden vier Wörtern: ö $\ddot{a}_{0}(s i \ddot{i}$ (nach einem anderen Gewährsmann ösi)

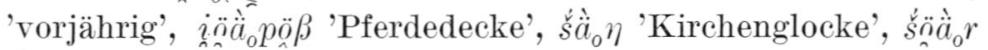
'runder Schleifstein' (s. Kannisto o. c. S. 24, 58, 69, 187, 188); in den drei letzten Beispielen ist der vordere Vokal offenbar durch die Einwirkung des mouillierten Konsonanten bzw. des $i$ bedingt. Auch das in Rede stehende Wort tššn, ši usw. hat einen mouillierten Konsonanten, aber es lässt sich nicht denken, dass die interdialektische Vokalserie $\varepsilon, \ddot{o}, \ddot{i}, i, i$ infolge einer Palatalisierung, die in den verschiedenen Mundarten getrennt stattgefunden hätte, aus einem früheren *a, * $a$ bzw. *o entstanden wäre, sondern das Wort muss schon von Anfang an vordervokalisch gewesen sein. Dem Umstand wiederum, dass die Bedeutungen der wogulischen und tatarischen Wörter ziemlich genau einander decken, darf man bei der Betrachtung der Frage nach der Entlehnung keinen grossen Wert beilegen. Im Wogulischen ist die Bedeutung 'Zeit' deutlich alt, und aus ihr haben sich die anderen Bedeutungen: 'ein gewisses Mass, eine gewisse Menge' und 'Art' leicht entwickeln können; bei der Entstehung der Bedeutung 'Mass' haben Fälle wie z. B. soat-si 'eine Woche' (s. oben), in welchen sich 'si an 'einen bestimmten Zeitabschnitt' bezeichnende Wörter angeschlossen hat, eine Zwischenstufe gebildet. Ein Beispiel für eine junge Entwicklung 'Zeit' > 'Mass', 'Art, Weise' bietet das folgende wogulisch-ostjakische Wort: wog. (Kann. Wog. Volksd. I, S. XLII, 454 und mscr.) KM $l \dot{o} \dot{a}^{\prime} t$ 'Zeit, Mal, Stunde', nə

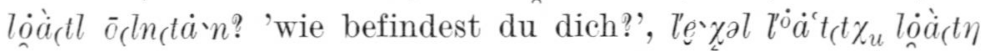
kinm 'gesprächiger Mensch'; ostj. (Karj. -Toiv.) DN tàt, țàt,

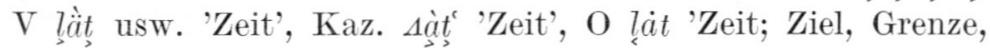
"Zielstreifen»', tàm látna ' 'tähän asti (ei ajassa)' [d.h. 'bis

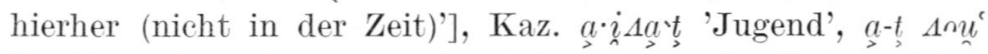
'keskikokoinen hevonen (ei suuri eikä pieni)' [d. h. 'mittelgrosses Pferd (nicht gross und auch nicht klein)'], O a iz a $\dot{a} t=$

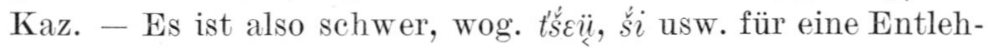
nung aus dem Tatarischen zu halten, und so hat Kannisto es 
denn auch nicht in sein ausführliches Werk »Die tatarischen Lehnwörter im Wogulischen» aufgenommen. Mit gutem Grund kann man das Wort demnach für bodenständig halten.

Hier muss man aber auch das folgende ostjakische Wort in

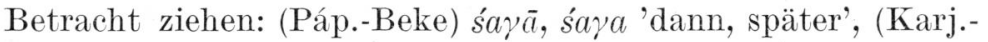

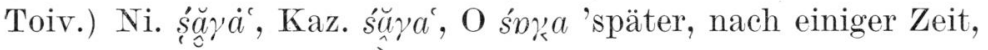
nachher', (Stein. Ostj. Gramm.) Scher. śă $a$ 'dann'. Dieses erinnert lautlich an das wogulische Wort, aber trotzdem bleibt unsicher, ob es mit ihm etymologisch zusammengehört. W. Steinitz hat es nämlich in seiner ostjakischen Chrestomathie S. 90 und Grammatik S. 157 zu Scher. śăta, śăt 'dort, da', śătta, Li śălta 'von da; darauf, dann', šs, śìt 'der, dieser' gestellt. Die letztgenannten Formen lauten nach Karjalainen

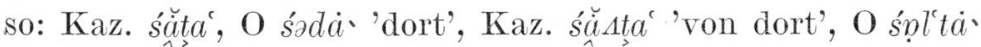
'dann', Kaz. śl̆ 'der dort, dieser', O śi 'der dort' usw. Nach Steinitz ist śă $\chi a$ also von einem Pronominalstamm gebildet. Semasiologisch passt das gut. Der Form nach müsste śă $a$, śă $\underset{c}{c} \gamma a$ usw. dann ein Lativ sein. Als Temporal dient im Ostjakischen allerdings der Lokativ auf -na (s. z. B. Steinitz Ostj. Gramm. 51), aber in einigen Fällen wird auch der Lativ als Zeitbestimmung gebraucht, z. B. (Karj.-Toiv.) Kr. at usw. 'Nacht': Kr. ai ‘்

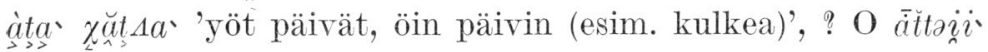
'yöllä' (in dem letzten Beispiel liegt, wenn es hierher gehört, ein $i$-Lativ vor); siehe auch F. KARA NyK XLI 160-161. Steinitz' Erklärung ist also möglich, und die lautliche Ähnlichkeit der ostjakischen und wogulischen Wörter scheint demnach nur auf Zufall zu beruhen.

Nach diesem Exkurs können wir wieder zu ung. -ság, -ség zurückkehren. Semasiologisch passt es, wenn man von der Grundbedeutung 'Zeit' ausgeht, gut mit wog. $t^{\prime s} s \ddot{z}$, š̉ usw. zusammen, und auch das Lautliche verursacht keine grossen Schwierigkeiten. Der Vokalismus ist ein ähnlicher wie z. B. in den bekannten Wörtern: wog. (Kann. Vok. 150) TJ, TČ, $\mathrm{P} l \varepsilon l t-$, KU, KM lalt-, KO lictt-, VN, VS litt- usw. 'atmen, aufatmen, seufzen' ung. lélek 'Seele, Geist'; wog. (Kann. Vok. 45)

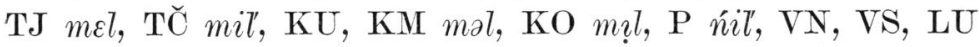
milla usw. 'tief' $\sim$ ung. mély. id. Der anlautende Konsonant 
war, nach dem Zeugnis des Wogulischen, die mouillierte Affrikata $* t s$. Im Ungarischen wird diese allerdings gewöhnlich durch $c s$ vertreten, aber in einigen Fällen begegnet auch $s$,

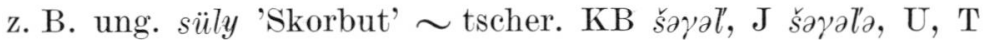

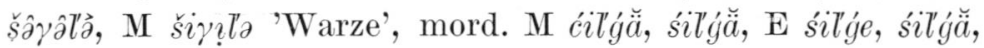
selǵă 'Warze, Hautpustel', fi. syylä 'Warze' usw. (s. z. B. Setälä FUF II 240, Wichmann FUF XI 188, 263, Szinnyei $\mathrm{NyH}^{7}$ 29). Verwickelter ist die Frage nach den auslautenden Konsonanten, denn wog. $\beta, \gamma$ geht zunächst auf $* \gamma$ zurück, ung. $g$ wiederum auf $* \eta k$. Dieser Widerspruch gleicht sich jedoch aus, wenn man eine gemeinsame Urform * $\eta$ annimmt. Dabei muss man aber die Vertretung des * $\eta$ in den ugrischen Sprachen einer näheren Betrachtung unterwerfen.

Das $\eta$ hat in den ugrischen Sprachen bekanntlich eine zweifache Vertretung, nämlich: 1) ung. $v$, ø, wog.-ostj. $\eta, \gamma$, $\beta, u, \varnothing$ und 2) ung. $g$, wog.-ostj. $\eta k, \eta k_{\varepsilon}$. Ung. $g$ und wog.-ostj. $\eta k$, $\eta k_{\varepsilon}$ sind eigentlich die Fortsetzungen des fiugr. $* \eta k$, und die Wörter, die sie aufweisen, sind also in die Gruppe der $* \eta k$-Wörter übergegangen. In einem Teile der Wörter ist die Vertretung in allen drei Sprachen einheitlich, z. B. ung. nö 'Weib, Frau' wog. (Kann. Vok. 41) TJ, TČ $n \bar{\imath}, \mathrm{KU}, \mathrm{VN}$

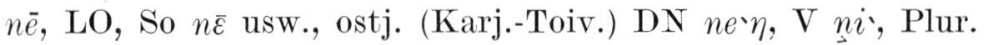

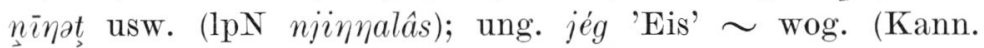

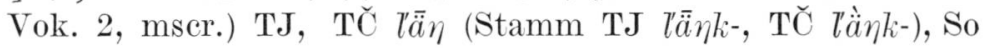

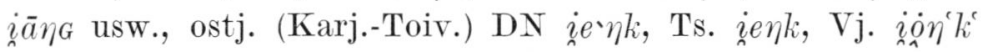
usw. (fi. jää); ung. egér 'Maus' wog. (Kann. FUF XXI 37)

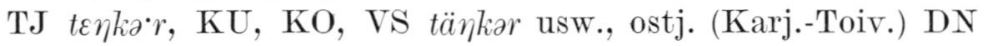

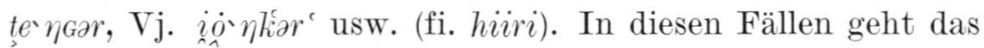
* $\eta k$, wie man erklärt hat, auf die gemeinsame urugrische Grundform zurück. Aber in einem anderen Teile der Wörter wechseln die zwei Typen der Vertretung miteinander. So entspricht dem ung. $v$, ø wog.-ostj. $\eta k$ in den folgenden bekannten Fällen: ung. fö 'Kopf, Haupt' wog. (Kann. Vok. 33, mser.) TJ, TC̆

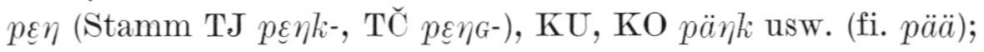

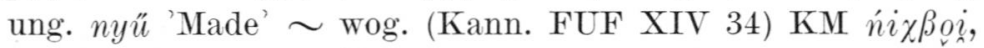

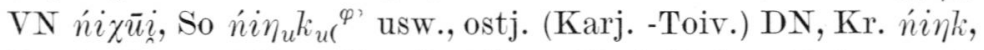
$\mathrm{V}$ nì' $k$ usw. Dasselbe lautliche Verhältnis würden auch folgende Wörter zeigen: ung. szöke 'blond', (in der alten Lite- 


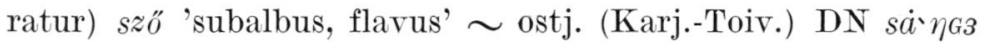
'hell, klar (Luft, Wasser); Licht, Helligkeit; Welt', V sä̀nki 'hell, klar (Tag, Wasser, Feuer); grell; scharf (Auge)' usw.1 (s. zu diesen Munkáesi NyK XLVII 462 und György LAkó NyK LI 171); ung. lóbál 'etw. schwingen, hin und her bewegen; schlenkern', lódul 'sich in Bewegung setzen; sich packen,

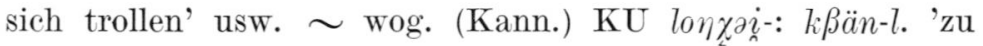
früh gebären (von der Frau)', syrj. ledni 'werfen; hinauswerfen; ausschöpfen usw.', wotj. ledyny 'schöpfen, ausschöpfen', tscher. $l o \cdot \eta g a m, ~ l o \eta a \cdot m$ 'wannen, worfeln; den Kopf zurückwerfen', mord. lijams: kotf $l$ '. 'ein Gewebe aufziehen', $1 p K$ loñni- 'heben', fi. luoda 'werfen, aufwerfen, ausschöpfen, erschaffen usw'. (s. Lakó NyK LI 165-173, der das ungarische Verb mit den übrigen, schon früher zusammengestellten Wörtern vergleicht). Diese beiden Zusammenstellungen sind jedoch unsicher. Hinsichtlich der ersteren ist nämlich zu beachten, dass man einerseits das ungarische Wort auch als eine slawische Entlehnung erklärt hat (s. Bárezi Szófejtő Szótár 294 mit Literatur) und dass man andererseits das ostjakische Wort auch mit fi. sä̈ 'tempestas, variatio tempestatis', 'Witterung' verglichen hat (s. zuletzt Toivonen Suomi V: 16, S. 305-306 und Steinitz Finn.-ugr. Vok. 41); bei der letzteren wiederum bereitet die Bedeutung des ungarischen Wortes einige Schwierigkeiten.

Einen entsprechenden Wechsel $\gamma, \beta, u, \eta \sim \eta k, \eta k_{<}$trifft man auch in den Ob-ugrischen Sprachen an. Lakó, der die Vertretung des $* \eta$ zuletzt eingehender behandelt hat, führt in NyK LI 171-172 folgende zwei Beispiele dafür an: wog. tn $\beta$ 'Schemen- oder Schattenseele des Menschen' $\sim$ ostj. ton $\chi$

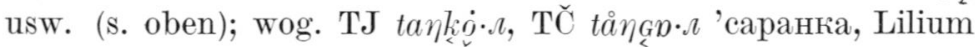

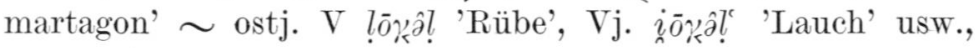
samK t'ù นฺ 'Lilienzwiebel (von Lilium martagon)' (zusammengestellt von Toivonen FUF XXI 119-120; nach ihm

1 Das von Munkácsi und Lakó erwähnte wog. sāykali, sānkamli 'teremtődni; felébredni', sā $k_{k} m t i$ 'teremteni' ist von dem ostjakischen Worte zu trennen, denn es gehört offenbar mit ostj. (Karj.-Toiv.)

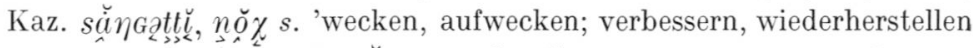

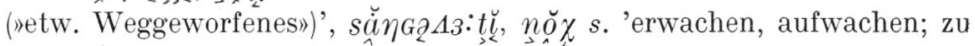
sich kommen usw.' usw. zusammen. Wog. < ? Ostj. 
Sam. < ? Ostj.). Das Verzeichnis solcher Wörter lässt sich leicht vermehren, z. B. ostj. (Karj.-Toiv.) DN tṣ̆iu, V tșs̀u, Ni.

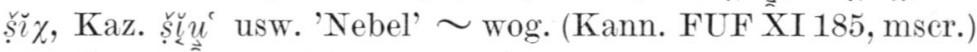

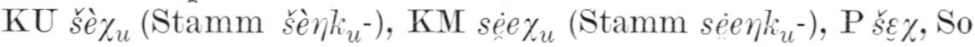
sē $\eta G^{\varphi}$ usw. id., syrj. tșin 'Rauch', wotj. tṣ̌̂ Wichmann FUF XI 185 mit Literatur und Uotila Syrj. Chrest. 164); wog. (Munk.-Szil., NyK XXIII 87) P owèt, LM äw, N àwa 'Kopf des Bären', (Kann. MSFOu. LII 103, mscr.)

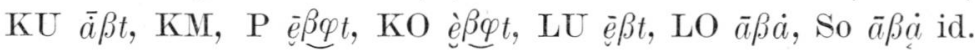

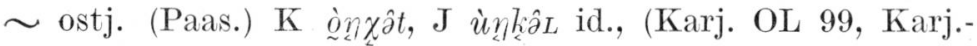

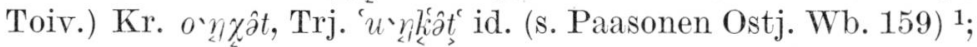
wog. (Munk.-Szil.) T lèn, P lèin, LM liin, $7 \bar{i} n, \mathrm{~N}$ lè in 'Eich-

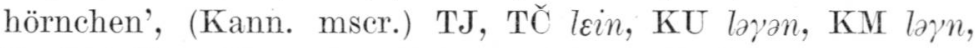

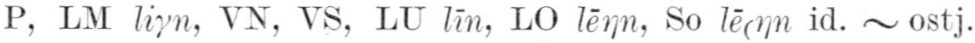

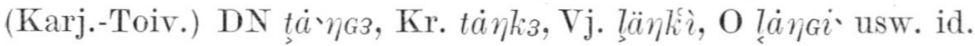

Wie Lakó in NyK LI 171-173 hervorhebt, beweist ein derartiger Wechsel in der Vertretung des * $\eta$, dass der Übergang der * $\eta$-Wörter zu den * $\eta k$-Wörtern nicht immer einheitlich auf dem ganzen urugrischen Sprachgebiet stattgefunden hat, sondern oft nur dialektweise vor sich gegangen ist. Wahrscheinlich ist die *nk-Vertretung nicht in allen Fällen gleich alt.

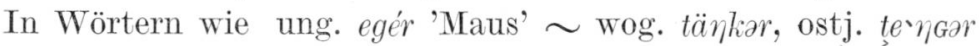
(s. oben), in denen sie in allen drei Sprachen erscheint, kann sie schon früh aufgekommen sein, während sie dagegen in den Beispielen, in welchen sie nur in einer bzw. zwei Sprachen vorkommt, sicherlich erst aus der späturugrischen (oder aus einer noch jüngeren) Zeit stammt. Im Ostjakischen und dialektweise auch im Wogulischen ist das * $\eta$ in intervokalischer Stellung in einigen Fällen noch bewahrt; so verhält es sich

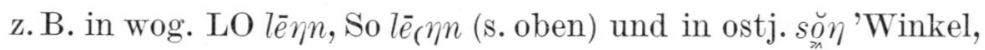
Ecke' (s. weiter unten). In der ursprünglichen Verbindung * $\eta k$ ist der Nasal in den beiden Sprachen allgemein erhalten.

1 Eine andersartige Erklärung des wogulischen Wortes bei Munkácsi NyK XXIII 87, 345, Kannisto MSFOu. LII 103-104, T Lehtisalo FUF XXI 19 und Dávid Fokos Nyr. LXVII 55. Nach J. Pápay, Északiosztják medveénekek, S. 129, 132, 134 kommt im Nordostjakischen auch ein Wort àuà 'a medve feje' vor. Es gehört, wie Pápay erklärt, mit wog. āsva zusammen, ist aber augenscheinlich diesem entlehnt. 
Offenbar war dann das $* \eta$ in den beiden Stellungen noch in der späturugrischen Zeit bewahrt, und zwar auch in dem urugrischen Dialekt, aus dem das Ungarische hervorgegangen ist. Voraussetzungen zum Übergang einzelner * $\eta$-Wörter in die Gruppe der * $\eta k$-Wörter waren also wenigstens noch damals in allen drei Dialekten vorhanden, da aber die urugrischen Bevölkerungsgruppen zu dieser Zeit nicht mehr in fester Verbindung miteinander standen, konnte sich das * $k_{k}$ nicht in allen Fällen über das ganze ugrische Sprachgebiet ausbreiten. Auf diese Weise wird verständlich, warum z. B. in dem Worte ung. nyи $\sim$ wog. nixßoi, ostj. nink (s. oben) die *nk-Vertretung nur auf der $\mathrm{Ob}$-ugrischen Seite begegnet. Da es sich aber damit so verhält, muss auch der Gedanke möglich sein, dass einige Wörter nur in der späturugrischen Mundart, aus der das Ungarische entstanden ist, oder vielleicht noch im Vor-

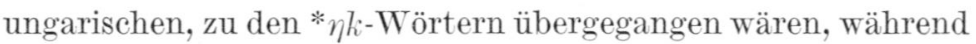
im Wogulischen und Ostjakischen eine Entwicklung * $\eta>\gamma$, $\beta, u$ usw. stattgefunden hätte. Es gibt in der Tat einige Beispiele, deren Konsonantismus sich am leichtesten gerade durch Annahme einer solchen Entwicklung erklären lässt. Dies sind:

1. Das ungarische Verb fog 'fangen, fassen, greifen; etw. beginnen, zu etw. schreiten; einspannen, spannen'. Schon Budenz hat es in MUSz. 526-529 u. a. mit folgenden Wörtern zusammengestellt: wog. (Kann. Vok. 170, 121) TJ $p \dot{o}^{\beta}-$, T $\breve{O}$ $p_{\llcorner}^{\dot{\rho} \beta-}, \mathrm{KU} p \dot{\rho} \beta-, \mathrm{KM} p \bar{u} \beta-, \mathrm{KO} p u \check{\beta} \beta-, \mathrm{P}, \mathrm{VN} p u \beta \beta-, \mathrm{VS}, \mathrm{LU}$

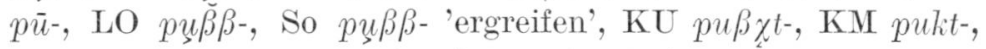
$\mathrm{P}$ pùkt-, VN pūkt- usw. 'umfassen, landen', mord. E pongoms, M povâms 'geraten, hineingeraten, stecken bleiben; ergriffen werden; treffen; sich treffen', est. pōma 'aufhängen, henken; (intrans.) hängen'; ung: und mord. g wären nach ihm Ableitungssuffixe: ung. fog $<{ }^{*} f o v o g$. Szinnyei (s. z. B. NyH ${ }^{7}$ 65) vergleicht nur die ungarischen und wogulischen Wörter miteinander und hält, ebenso wie Budenz, ung. $g$ für ein Suffix; der Stammkonsonant wäre demgemäss entweder frühurugr. *k oder * $u$ gewesen. Auch Gombocz und Melich in MESz. II 316 sowie Bárczi in Szófejtő Szótár 81 verbinden ausschliesslich die ugrischen Wörter miteinander, halten aber ihre Zusammengehörigkeit für unsicher und die lautlichen Verhältnisse für 8-Finnisch-ugrische Forschungen 
unklar. Paasonen seinerseits stellt in seiner Mord. Chrest. 112, 156 die estnischen, mordwinischen und ungarischen Wörter zusammen und geht also offenbar von einem inl. * $\eta$ aus. Nach Uotila schliesslich könnten alle obenerwähnten Wörter und ausserdem noch wotj. pumîn ?̂ 'jemandem begegnen', puniśkini usw. 'sich begegnen', punit usw. 'gegen, entgegen', syrj. panid usw. 'entgegen' usw. etymologisch miteinander verwandt sein; der inlautende Konsonant wäre * $\eta$ gewesen, das im Wogulischen $\beta$, im Ungarischen dagegen $g$ ergeben hätte (s. FUF XXVI 166-169, Syrj. Chrest. 130, Vir. 1944, S. $32-33$ ). Von diesen verschiedenen Erklärungen erscheint vom Blickpunkt des. Ugrischen aus die von Uotila gegebene am glaubwürdigsten. Durch sie wird der inlautende Konsonant der ungarischen und wogulischen Wörter, die man wegen der identischen Bedeutung voneinander nicht trennen kann, vollkommen klar. Dagegen bleibt es unsicher, ob die anderen angeführten Wörter hierher gehören.

2. Auch im folgenden Substantiv entspricht dem ung. $g$ in den Ob-ugrischen Sprachen $\beta$, u: ung. tegez 'Köcher' $\sim$ wog. (Munk.-Szil., VNGy. II 0548) K, LM täut, N taut, tawèt id.,

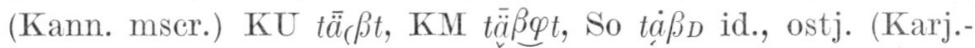

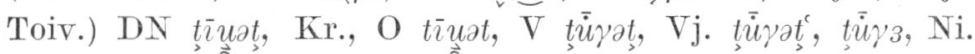
ț̌rat usw. id. (s. z. B. Budenz MUSz. 200, Szinnyei NyH? 146 und über die ethnographische Seite Kustaa Vilkuna MSFOu. XCVIII 376-). Die Ansichten über den Ursprung dieser Wortsippe sind jedoch nicht völlig übereinstimmend. Munkácsi nämlich hat, und zwar gerade wegen der inlautenden Konsonanten, die Wörter für entlehnt gehalten; sie wären getrennt aus dem Worte übernommen, das dschag. tigdi 'Sack von Leder od. Filz' vertritt, oder aber es läge hier eine Entlehnung aus dem Arischen vor, wo sanskr. dhākás, dhāká'Behälter' das Original verträte (s. ÁKE 594-596). Auch M. ZsIRAI sieht hier ein arisches Lehnwort, obgleich er kein Original anführt (s. Finnugor rokonságunk, s. 148 und das von LaJos Ligetr redigierte A magyarság őstörténete, S. 32). Dieser Name des Köchers kam sicherlich schon in der urugrischen Grundsprache vor; das beweisen die auslautenden Konsonanten ung. -z, $\sim$ wog.-ostj. -t, die ähnlich sind wie 
z. B. in den Wörtern ung. kéz 'Hand' wog. (Kann. Vok. 3)

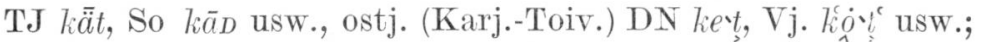

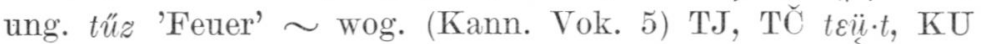

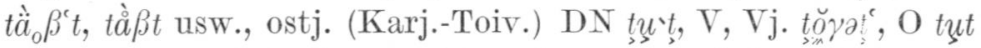
usw. Hier im Wortinnern kann das ung. $g$ kein Ableitungssuffix sein, und andererseits lässt sich ung. $g \sim$ wog. $\beta$, ostj. $u$ nicht aus einem urugrischen Palatalklusil herleiten. Der inlautende Konsonantismus wird dagegen vollkommen klar, wenn man annimmt, dass das vorliegende Wort in der urugrischen Grundsprache * $\eta$ hatte und dass dieses im Ungarischen zu $\left(>*^{*} \eta k\right) g$, im Wogulischen und Ostjakischen dagegen zu $\beta, u$ wurde.

3. Ein solches Wort ist ferner ung. zug (in der alten Literatur auch szug) 'Winkel, Ecke', szugoly id., mit dem schon seit Budenz ostj. (Karj.-Toiv.) DN, Kr. O sŏn, V, Vj., VK, Trj. sŏn', Ni., Kaz. sŏy' usw. 'Winkel, Ecke usw'., Stein. (Ostj. Chrest.) suฑ 'Ecke', (Ostj. Gramm.) sŭ worden ist. Die Wörter passen in jeder Hinsicht gut zusammen. Der ostjakische Vokal geht auf einen o-Laut, nach Karjalainen auf $*_{o}$ (s. OL 287), nach Steinitz auf *ŏ (s. Ostj. Vok. 85) zurück. Beispiele zu der Entsprechungsproproportion ung. $u$ $\sim$ ostj. ŏ usw. s. z. B. bei Steinitz Finn.-ugr. Vok. 21-25. Ostj. $\eta$ ist alt, ähnlich wie z. B. in dem Worte (Karj.-Toiv.) DN, Kr. tŏ $\eta$, Koš., Sogom, Fil. tū 'Sommer' usw. (in allen Dialekten $\eta) \sim$ fi. suvi. Es ist aber zu beachten, dass im Ungarischen auch ein gleichbedeutendes vordervokalisches Substantiv vorkommt, näml. szög, szeg 'Winkel, Ecke'. Einige Forscher haben das letztere nur für eine Nebenform des ersteren gehalten. So vergleicht Paasonen Beitr. 30, 222 ausser zug, szug auch szög, szeg mit dem obenerwähnten ostjakischen Worte und ferner mit sam. Jur. siejea, O seänä 'Winkel, Ecke', und auch Steinitz Ostj. Gramm. 157 führt, allerdings mit einem Fragezeichen versehen, als Entsprechung von ostj. sŭ alle drei ungarischen Formen an. ${ }^{2}$ Die ungarischen Forscher

\footnotetext{
1 Wog. sām 'Gegend, Ecke, Winkel', das Paasonen auch anführt, gehört mit keinem der genannten Wörter zusammen.

2 In Ostj. Chrest. S. 90 erwähnt Steinitz bei ostj. suך nur die vordervokalischen ungarischen Wörter, und auch Setälä FUF XII Anz. 21
} 
dagegen haben allgemein das hintervokalische Wort von den vordervokalischen getrennt. Die letzteren haben sie auf mehrere Weisen erklärt: in ihnen hat man einen Vertreter des Verbalstammes szeg 'frangere, rumpere, proscindere', eine Nebenform von szeg 'Nagel, Stift' und eine Entlehnung aus slaw. stbgz, vgl. z. B. kirchenslaw. stbgna 'angulus, vicus' gesehen (s. Bárczi, Szófejtő Szótár 294 mit Literatur). Wie die ungarischen und samojedischen vordervokalischen Wörter letzten Endes auch zu deuten sind, ung. zug, szug und ostj. sŏn, sŏ $\eta^{c}$ kommen jedenfalls lautlich und bedeutungsmässig einander so nahe, dass man sie kaum voneinander trennen kann, und so bieten sie ein Beispiel, in welchem der Übergang $* \eta>* \eta k$ nur im Ungarischen begegnet.

4. Ein Beispiel für einen nur im Ungarischen vor sich gegangenen Übergang eines $* \eta$-Wortes zu den $* \eta k$-Wörtern bietet möglicherweise auch ung. mögött 'hinter', s. darüber' gleich unten.

Die obenangeführten Beispiele beweisen, dass ung. $g$ und wog. $\gamma, \beta$ in einzelnen Fällen auf denselben urugrischen Laut * $\eta$ zurückgehen können. Die Zusammenstellung ung. -ság, -ség $\sim$ wog. t'śri, š́ usw. ist demnach auch hinsichtlich der auslautenden Konsonanten vollkommen möglich.

\section{Ung. mögött wog. mänt.}

Ung. mögött 'hinter (mit dem Dativ)', mögé 'hinter (mit dem Akkusativ)', mögül 'von hinten hervor, von hinten her' hat bekanntlich in den verwandten Sprachen folgende Entsprechungen: syrj. miśt, miśs 'nach (von der Zeit)', tscher. mə•ngə,

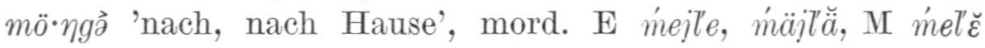

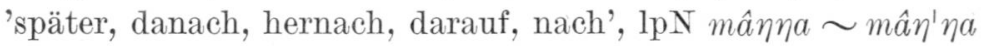
'afterwards, later, after (in time) usw.', fi. myöden, myöten 'längs, gemäss, nach'. Der inlautende Konsonant war also $* \eta$, und im Ungarischen ist es durch $g$ vertreten. Diese Wort-

und JSFOu. $\mathrm{XXX}_{5} 31$ stellt aus dem Ungarischen nur szög, szeg zu den ostjakischen und samojedischen Wörtern. 
sippe scheint aber einen Vertreter auch im Wogulischen zu haben, obgleich beim ersten Anblick allerdings in einer auffallenden Form, näml. (Munk.-Szil.) K mänt, mäntèl 'längs, durch, während', P mäntèl (॰: mäntèl, s. NyK XXIV 18) 'durch', LU mańtèl 'in der Richtung', üt'-aš m. 'a víz fölszíne mentében', LM mäntä, mänti, mäntèl 'längs, unterdessen', (NyK XXIII 377, 381) K lōñ kh-mäntèl jōmi 'az út hosszában jár', kwọ̈snäsmäntèl 'az ablakon keresztül', am tèt vọnlènèm-mäntël 'mialatt én itt ülök' usw.; (Kann. FUF XXVI 202) KU mänt, mänts,

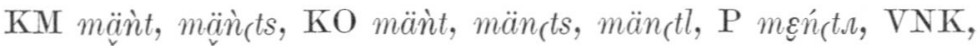

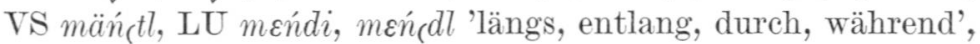

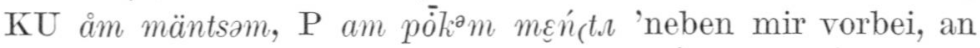
mir vorbei', (Wog. Volksd. I 386) ńassnå̀m die Nasenlöcher'.

Semasiologisch ist die Zusammenstellung anstandslos. Im Wogulischen hätte nur eine ähnliche Bedeutungsentwicklung stattgefunden wie in fi. myöden, myöten oder in dem wogulischen Worte (Munk.-Szil., NyK XXIV 314) T sëi, sai 'entlang',

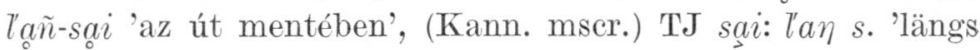
des Weges' $\sim$ (Munk.-Szil.) N saj 'abseits gelegener Ort', sajit 'hinter', K saịi 'mögé, mögött' usw. Auch das Lautliche bereitet keine grossen Schwierigkeiten. Erstens ist nämlich zu beachten, dass die interdialektische Vokalserie KU, KO, VN, VS $\ddot{a}, \mathrm{KM} \ddot{a}, \mathrm{P} \varepsilon, \mathrm{LU} \varepsilon$ oft einen vorderen Labialvokal vertritt. Da fi. myö- nach ERKKI ITKONEN auf *münä zurückgeht (s. FUF XXX 39), ist die Entsprechungsproportion der Vokale in dem vorliegenden Falle eine ähnliche wie z.B. in dem Worte wog. (Kann. Vok. 30) KU, KO, VN, VS täl, KM täl, P tel', LU $t \varepsilon l$ usw. 'Faden (Mass)' $\sim$ ostj. (Karj.-Toiv.) DN tat, Vj.

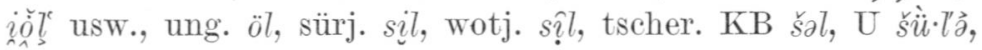
mord. E sęl', säl', M śel', lpN sâllâ, fi. syli (zu dieser Vokalvertretung s. Steinitz Finn.-ugr. Vok. $25-28$ und Toivonen FUF XXIX 160-167). Verwickelter ist der Konsonantismus, und er bedarf einer näheren Betrachtung.

Das auslautende $-l$ ist die Endung des alten Ablativs, das $-\ddot{a},-i$ wiederum die des Lativs. Das Wort mänt, mäntèl usw. hat eine prolativische Bedeutung, und in dieser Funktion wird häufig gerade der Ablativ auf -l, -nal angewendet (s. z. B. 
ÖDÖN BEKE NyK XLI 250-251), daneben aber auch der Lativ, z. B. (Munk.-Szil., NyK XXIV 312, 313) T khošto 'entlang', lañ-khošto 'az út hosszában'; $\mathrm{N}$ au-sisi' 'in der Richtung des Stromes', T $\bar{u}$-šr $i s ̌ u$ id. ( sis, š šs 'Rücken'). Trotz alledem sind die Endungen $-l$ und $-\ddot{a}$, $-i$ sowie das Element $-s$ hier wahrscheinlich sekundär. Die Konsonantenverbindung nt lässt sich nämlich in dem in Rede stehenden Worte leicht als sekundär erklären, und das $t$ dürfte dann das Suffix des Lokativs sein. Es ist zu beachten, dass der $t$-Lokativ im Wogulischen ganz üblich ist; er ist der einzige lokativische Kasus der heutigen Nominaldeklination. Und auch dieser Kasus wird in der Funktion des Prolativs angewendet, z. B. (Kann.) KM

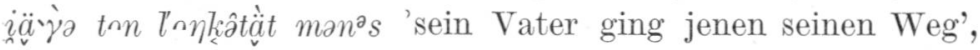
So iāßātàt iñmi 'sie geht am Ufer eines Flusses hin' (s. Kannisto Wog. Volksd. I 55, 220), (mscr.) So āzt ta mi ni 'er fährt

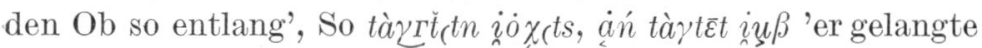
zu der Sosva, der Sosva entlang kommt er', KM êj i iuß tu's 'er trat durch die Tür hinein', (Wog. Volksd. I 110) KM so'-

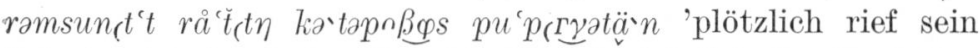
Schutzgeist durch das Rauchloch'. Hinsichtlich der Funktion kann mänt also Lokativ sein. Erst nachdem es nicht mehr als obliquer Kasus aufgefasst wurde, nahm es die Endungen -l, $-\ddot{a},-i$ und das Element $-s$ auf; dies hat schon ziemlich früh stattfinden können. Der Stamm des Wortes hätte also *mäna

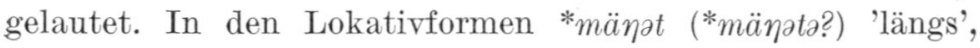
*mänatam 'längs mir, an mir vorbei' usw. sowie wohl auch in

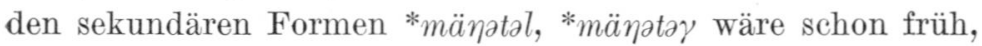
offenbar schon in der späturwogulischen Zeit, der Vokal der zweiten Silbe geschwunden. Eine alte Synkope trifft man im Wogulischen auch sonst, u. a. in den Lativen, die die Endung $-\gamma \partial,-\gamma \dot{a}$ usw. aufweisen, z. B. (Kann. mscr.) 'TJ $\varepsilon l k_{<}^{u} i \cdot l^{\prime}, \varepsilon l \chi_{u}$,

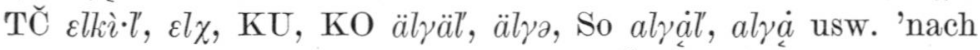
dem Oberlauf des Flusses' ( TJ, TČ $\varepsilon_{(} l n \cdot$, KU $\vec{a}_{C} l n$ usw. 'am Oberlauf des Flusses'); vgl. dazu die Endung $-\AA a \beta,-\varepsilon \gamma,-i$

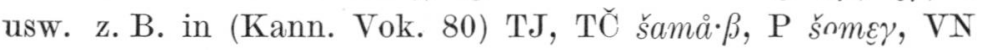
šnmi, LU šamā usw. 'steil, aufrecht, schroff' (s. auch Liimola FUF XXX 277-278). In den synkopierten Formen *mä *mäntam usw. wäre das * $\eta$ unmittelbar vor $t$ dann zu $n$ gewor- 
den. Eine ähnliche Entwicklung * $\eta t>n t$ hat nämlich in folgenden Wörtern stattgefunden, obzwar die Verbindung * $\eta t$ in ihnen allerdings alt ist: 1) wog. (Munk.-Szil.) LO int keer 'Spindelring', (Kann. mser.) TJ iznt, TČ icìt, KU izant, KM iaǹt, KO itìnt, $\mathrm{P} i \grave{n}(t, \mathrm{VNK}$ iǹt, VS, LM $i n t$, LU (nach versch. Gewährsmännern) int, int 'Spindel' ostj. (Karj.-Toiv.)

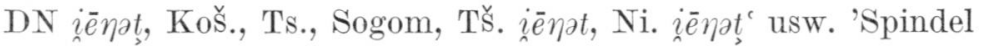
usw.'; 2) wog. (Munk.-Szil.) K, LM kèt, N kēnt 'Mütze', (Kann. mscr.) KU kènt, LO kēnnt, So kèēnd id., KM keèe't id., Plur.

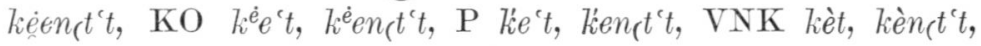
VS ke't, kenst't, LU kę $t$ id., kend ${ }^{2} m$ 'meine Mütze' $\sim$ ostj. (Karj.-Toiv.) DN, DT kanat, Koš., Kr. kanat 'Mütze'; 3) wog. (Munk.-Szil.) T änt, N ānt 'Horn', K ōntéẽ 'aus Horn', (Kann.

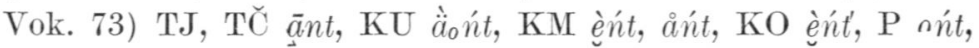
$\mathrm{VN}, \mathrm{VS}$ ańt, LU (von versch. Gew.) ańt, ånt, LO ānt, So āñ

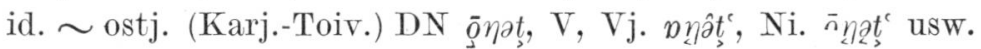
id. ${ }^{1}$ In den angeführten Beispielen hat der Wandel * $\eta t>n t$ offenbar schon in der späturwogulischen Zeit stattgefunden, aber im folgenden Wort erst, nachdem die Sonderentwicklung der Dialekte schon angefangen hatte: wog. (Munk.-Szil.) N

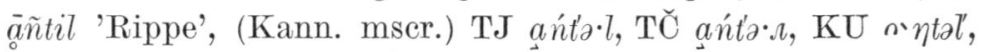

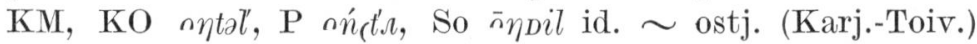

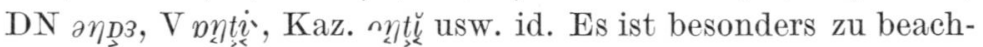
ten, dass man in dem wogulischen Worte mänt von * $\eta$ und

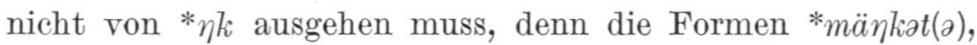

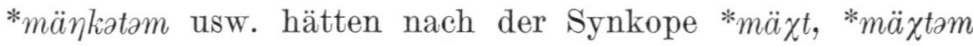
usw. ergeben. - Auch das Wort wog. mänt ung. mögött gehört also, wenn sich die Zusammenstellung als richtig erweisen wird, zu den Fällen, in denen der Übergang $*_{-} \eta->*_{-} \eta k$ - in den ugrischen Sprachen nur im Ungarischen vor sich gegangen ist.

Früher hat der Unterzeichnete wog. mänt mit $\mathrm{lpN}$ mod'del $\sim$ mod'dèl 'past (and going on)' usw., moed'det 'miss (not hit); mistake something, e. g. one's way' usw. zusammengestellt (s. FUF XXVI 201-204). Erkki Itkonen und Toivonen wiederum

${ }^{1}$ Mit diesem Wort hat man sam. Jur. ńamd, ńämd 'Horn', T namta, Jen. eddo usw. id. zusammengestellt, die ein altes *-mt- enthalten. Siehe I. HalÁsz NyK XXIV 453, Setälä FUF XII Anz. 78 und Paasonen Beitr. $2 \dot{b}$. 
haben das lappische Verb mit ostj. (Karj.-Toiv.) V mìnto $\chi_{\rightarrow} t$ ă $^{\circ}$ ',

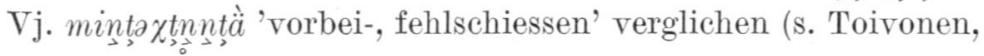
Sitzungsberichte der Finnischen Akademie der Wissenschaften 1949, S. 177). Es wäre nun nicht unmöglich, wog. mänt mit diesen beiden Wörtern zu verbinden, aber die oben gegebene Erklärung ist semasiologisch und hinsichtlich des Vokalismus wahrscheinlicher.

Zum Schluss muss man hier noch kurz ein ostjakisches Wort betrachten, näml. (Castr.) megde 'vorüber, vorbei', (Patk.)

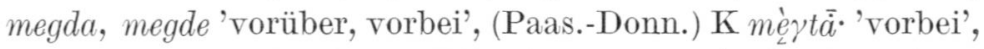
(Karj.-Toiv.) DN mè 'vorbei, vorüber'. Dieses erinnert in gewissem Mass an das oben behandelte wog. mänt, mäntèl, und wenn sein $\gamma$ auf ein früheres $* \eta$ zurückginge, liessen sich die zwei Wörter in der Tat lautlich verbinden. Ostj. - $\dot{a}$, usw. ist die Endung des Lativs, und da der $t$-Lokativ in dieser Sprache selten ist er begegnet nur in einzelnen Adverbien -, kann das $t$ keine Kasusendung sein, sondern es gehört offenbar zum Stamm. Dann müsste man das Ob-ugrische Wort von ung. mögött und dessen Sippe trennen. Die hier angedeutete Erklärung wäre jedoch ganz unsicher. Erstens ist nämlich zu beachten, dass bei dem wogulischen Worte die Bedeutung 'vorbei' jung' ist und nur in gewissen Wendungen vorkommt; das wogulische und das ostjakische Wort decken sich also semasiologisch nicht. Zweitens ist ostj. $m \grave{\gamma} \gamma_{\underline{D}} \dot{a}$ lautlich vieldeutig. Über es liegen Angaben nur aus solchen Dialekten vor, in denen die alten unmouillierten Sibilanten und das $* l$ beide durch $t$ vertreten sind. Sein $\gamma t$ könnte also auch auf *kl bzw. *lk zurückgehen. Und in Wogulischen gibt es denn auch ein anderes, ein $l k$ enthaltendes Wort, das semasiologisch viel besser als mänt,

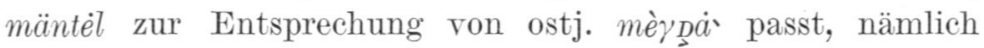
(Kann. mscr., Folkl.) KM mällkt 'vorbei, vorüber, gerade': täß m. man sta 'er ging an ihm vorbei, мимо ушель', in $\beta{ }^{\circ} \dot{a}^{\prime} t$ kioiraləsam, mälkt manasam 'ich kehrte nicht ein, (sondern) ging vorbei, мимо ушель', mälkt mәn $s$ 'er ging gerade, прямо ушель'. Das ostjakische Wort scheint also der Zusammenstellung wog. mänt, mäntèl $\sim$ ung. mögött usw. nicht im Wege $\mathrm{zu}$ stehen. 


\section{Syrj. laik.}

Über dieses Wort und seine Sippe bietet Yrjö Wichmanns Syrjänischer Wortschatz folgende Angaben: S, L laik munn 'sich biegen (infolge Druckes, z. B. First)'; V, I, U laikan 'Wiegenstange (die Wiege ist an das eine Ende derselben gehängt)'; U laike.dnis (tr.) 'schaukeln, wiegen'; V, L, I, U laikid 'schwankend, schaukelnd, biegsam', I l.-in 'Tal'; V laikja 'mit einer Einsenkung, sattelförmig' (z. B. Pferderücken, Gefäss)', S, L l.-koska (Adj.) 'mit einem Senkrücken (z. B. Pferd)', (auch) = lainkja; S, L, Peč lainjalni, V laikiavni, U laikja.vnis 'schwanken, wanken (z. B. Sumpf, Fussboden)' (S), 'schwanken, sich hin und her bewegen' (L, V), 'schaukeln (Peč: auf einer Zaunstange, U: auf einer Stange, einem Brett)'; S, Peč lainkjan = lainkan (Peč: das eine Ende der Wiegenstange ist schräg an der Decke befestigt), $\mathrm{P}$ vat'ta $n$-śor $(<*$ *aikja $n$ śor) 'Wiegenstange; Brunnenschwengel'; S laikjedlini.schwanken machen'; s laikizd = laikid: V, L laiknitni 'biegen, niederbiegen (z. B. das eine Ende der Stange, deren anderes Ende befestigt ist)'; U laikśinis 'sich biegen (Baum im Winde)'. In seiner Syrjänischen Chrestomathie S. 108 stellt Uotila zu diesem noch wotj. laikan 'Wiegenstange, Tragbaum, Stange' (s. auch Wichmanns Wortschatz s. v. laik). Wiedemanns und Munkácsis Wörterbücher kennen dagegen das wotjakische Wort nicht.

Was das Lautliche betrifft, ist das $k$ allem Anschein nach ein Ableitungssuffix; der Grundstamm lautete also *laj-. Sonst aber ist das $k$ mir nicht ganz klar. Syrj. laik ist ein Nomen, und von den übrigen Formen sind die meisten deutlich denominale Ableitungen. Syrj.-wotj. laikan scheint dagegen das bekannte Verbalnomen auf -an zu sein (im Wotjakischen wäre es aus einem Verb *lainkàn gebildet, s. Wiedemann Syrj. Gramm. 48 und K. Medveczky NyK XLI 431). ${ }^{1}$ Von dem in Rede stehenden Worte hätte demgemäss ein und derselbe

${ }^{1}$ In Wiedemanns Grammatik S. 47 ist syrj. laikan in der Tat als Beispiel für die mit -an gebildeten deverbalen Nominalableitungen angeführt. 
Stamm *laik- (vielleicht schon auch der Grundstamm *laj-) einmal sowohl als Nomen wie auch als Verb fungiert. Derartige Fälle sind ja in den finnisch-ugrischen Sprachen nicht selten; hier werde als Beispiel davon nur das syrjänische Wort ńum, ńm- angeführt: (Wichm.-Uot.) V ńum munni 'улыбнуться, lächeln' 〜 U ńumnis 'Улыбаться, id.', P ńmja.śni id., $\mathrm{V}$ numjavni, U numja.vnis id. Das k könnte demnach entweder ein Verbalsuffix oder ein Nominalsuffix sein; zu diesem vgl. z. B. syrj. (Wied.) P pol'k 'Blase', (Wichm.-Uot.) I polk 'Blase, Hitzbläschen' $\sim \mathrm{P}$ pol' 'Blase' usw. (s. Uotila MSFOu. LXV 127), zu jenem syrj. (Wichm.-Uot.) S, L ńoikalni, V ńoikavni 'sich erweitern, sich ausdehnen, sich dehnen (Kleider durch Tragen)' $\sim$ S, L, I ńoídni 'verfallen, zerfallen, auseinandergehen, sich abtragen, sich abnutzen (durch Tragen)'.

Für das permische Wort kennt man meines Wissens keine annehmbare Etymologie; Uotila z. B. führt in seiner Chrestomathie für es keine Entsprechungen an. Im Wogulischen gibt es jedoch ein Verb, das wahrscheinlich mit ihm etymologisch zusammengehört. Es lautet so: (Munk.-Trócs.) K laikwèsi 'biczeg', (Munk. VNGy. IV 400) tori-tol laikwessi 'daruláb

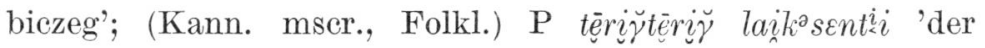
Kranich-Kranich schwingt sich hin und her'; KM (Folkl.)

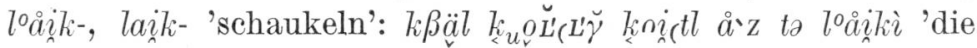
Hütte schaukelt sich ganz wie eine Sumpflache' (während Waldgeister darin toben), nårmə loain $k_{i}$ 'die Pritsche schwankt', $\beta о л \partial \gamma \sim \beta l$ saick $\chi$ 'ein Schaukelnder am Ende der Stange' (aus einem Rätsel, gemeint ist das Wiegen des Kleinkindes); KM (Folkl.) lo $\dot{a} i k$ lta $\chi_{c}$ ( $\chi_{u}$ 'sich wiegen (ein Baum)'; KM (Folkl.)

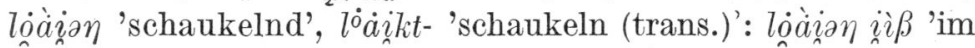

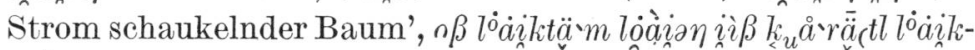
tn $\dot{\beta} \partial s \partial m$ 'nach Art eines vom Strom geschaukelten schaukelnden

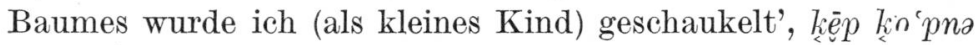
$l^{\circ} \dot{a} i_{k} t_{n} \hat{\beta}_{\partial}$ 'die Wellen schaukeln das Boot'. Die Vokale des Wortes gehen auf urwog. * $\bar{u}^{\mathrm{bzw}}{ }^{*} \varepsilon$ zurück, deren Fortsetzungen oft miteinander wechseln (s. Kannisto Vok. 1-, 16-, 131-). Der Grundstamm war also *läi- $\sim * l \varepsilon i$-. löàiza

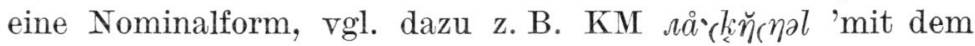

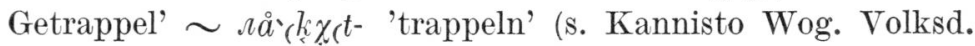


I 94), $\beta \ddot{a ̈}_{\gamma} \gamma \eta$ soi 'Geräusch (z. B. wenn die Enten fliegen)' $~$

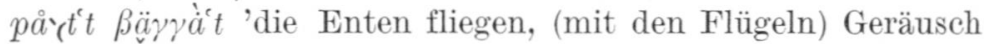
erzeugend' (o.c. S. 422) und s. ferner Munkácsi NyK XXIII 397 sowie D. Szabó NyK XXXIV 421-422. Das $k$ wiederum ist ein deverbales Verbalsuffix (s. Liimola FUF XXX 248-). Von den verschiedenen dem $k$ folgenden Suffixen sei hier nur das $\chi$ in.$a i d k \%$ hervorgehoben; es bildet Verbalnomina, in dem vorliegenden Falle ein Nomen agentis (s. Szabó NyK XXXIV 419-420). - Wahrscheinlich gehört hierher auch (Kann. mscr.) LO lài ся'. Das $\gamma$ ist ein Ableitungssuffix, so dass nur lài- als Stamm übrigbleibt, und dieses passt gut zu KM loài-, $l^{\circ} \dot{a} i-$, P lai-. Eine semasiologische Parallele bietet russ. (Pawlowsky) سamamb 'hin u. her bewegen, schütteln, rütteln, schaukeln', mamambcr 'sich hin u. her bewegen, wanken, schwanken, taumeln, wackeln; sich herumtreiben, müssig umherschlendern, bummeln usw.'.

Semasiologisch kommen das permische und das wogulische Wort einander ganz nahe. Was nun den Vokalismus betrifft, ist urwog. $* \bar{a}$, von dem hier auszugehen ist, die gewöhnliche Fortsetzung des fiugr. $* \ddot{a}$, und da auch syrj.-wotj. a dieses vertreten kann, ist die oben aufgestellte Etymologie auch lautlich möglich.

Matti Linmola. 